\title{
Liquid-liquid equilibrium of the Ucon 50-HB5100/sodium citrate aqueous two-phase systems
}

\author{
Gisela Tubio ${ }^{\mathrm{a}, \mathrm{b}}$, Bibiana B. Nerli ${ }^{\mathrm{b}, *}$, Guillermo A. Picó ${ }^{\mathrm{b}}$, \\ Armando Venâncio ${ }^{a}$, José Teixeira ${ }^{\mathrm{a}}$ \\ a IBB-Institute for Biotechnology and Bioengineering, Center for Biological Engineering, \\ University of Minho, 4710-057 Braga, Portugal \\ ${ }^{\mathrm{b}}$ Physical Chemistry Department and CONICET, Faculty of Biochemistry and Pharmaceutical Sciences, \\ National University of Rosario, Suipacha 570, S2002LRK Rosario, Argentina
}

\begin{abstract}
The phase diagrams of Ucon 50-HB5100/sodium citrate aqueous two-phase systems were determined at 5,20 and $40{ }^{\circ} \mathrm{C}$. Two medium pHs 5.20 and 8.20 were assayed. The binodal curves were satisfactorily described using a four-parameter sigmoidal equation. The two-phase area was expanded by increasing both $\mathrm{pH}$ and temperature. The reliability of the measured tie line compositions was ascertained by correlation equations given by Othmer-Tobias and Bancroft.
\end{abstract}

(C) 2008 Elsevier B.V. All rights reserved.

Keywords: Aqueous two-phase systems; Thermo-separating polymer; Binodal diagrams

\section{Introduction}

Aqueous two-phase systems (ATPSs) are formed when two mutually incompatible water-soluble polymers or one polymer and one salt are dissolved in water above a critical concentration $[1,2]$. These systems contain mainly water with a polymer predominating in one phase and the other polymer or salt predominating in the other. The high water content of both equilibrium phases $(>80 \%)$ provides a gentle environment for proteins, nucleic acids, viruses and other biological molecules. Therefore, the extraction technique using ATPSs is considered a powerful one since it allows the separation and purification of bio-molecules without losing their activity and structure [3,4]. In laboratory-scale separations the most commonly used systems are composed by the polymers polyethyleneglycol (PEG) and dextran while for large-scale enzyme extraction, PEG/salt systems are used. These systems are attractive because of their low-cost and rapid phase disengagement. In spite of that, the

Abbreviations: ATPS, aqueous two-phase system; EOPO, copolymer of ethylene and propylene oxides; PEG, polyethyleneglycol; Ucon, Ucon 50 HB5100; Cit, sodium citrate.

* Corresponding author. Tel.: +54 341 4804592; fax: +54 3414804598.

E-mail address: bnerli@fbioyf.unr.edu.ar (B.B. Nerli). high concentrations of salt (phosphate or sulfate) required to form the two-phase systems cause environmental problems. Previous studies [5] have demonstrated that replacing the inorganic salts by other biodegradable and non-toxic ones such as citrates could be considered a good alternative, since citrates can be discharged into biological wastewater treatment plants. However, the difficulty of removing the PEG from the product of interest, when this product has been selectively partitioned to the polymer rich-phase, is a remaining problem of these PEG/citrate ATPSs. A further processing of the top phase by ultra-filtration might be required thus compromising the potential commercial value of the product [6].

Recently, the use of thermo-separating polymers in ATPSs has been introduced [7]. When such polymers are heated above a lower critical solution temperature (LCST), the solubility of the polymer will decrease and a system composed of a water and a polymer phase is formed. This makes it possible to perform temperature-induced phase separation whereby a target protein can be separated from the polymer and recovered in the water phase. In addition, polymer can be recovered and recycled in the other phase. Many thermo-separating polymers contain ethylene oxide groups. PEG is also a thermo-separating polymer but its LCST is too high (above $100^{\circ} \mathrm{C}$ ) for separation of labile molecules. Several random copolymers of ethylene oxide 
and propylene oxide (EOPO) have LCST low enough to be applied for separation of biological molecules. This property makes these copolymers suitable as substitutes for PEG in the conventional polymer-salt systems.

In this work, Ucon $50 \mathrm{HB}-5100$, an EOPO random copolymer of 50\% ethylene oxide and 50\% propylene oxide (mass) with an average molecular mass of 3900 was selected to form ATPSs with sodium citrate at $\mathrm{pH} 5.20$ and 8.20. The corresponding phase diagrams were determined and the effect of temperature on the binodal curves was also studied.

\section{Experimental}

\subsection{Chemicals}

Ucon 50 HB-5100 (Ucon), a random copolymer of 50\% ethylene oxide and 50\% propylene oxide (mass) with an average molecular mass of 3900 was obtained from Union Carbide (NY). Citric acid was obtained from Sigma Chemical Co. and used without further purification. All the other reagents were of analytical grade with a minimum purity of $99 \%$. Enzymes for citrate determination were obtained from Boehringer Ingelheim, Germany.

\subsection{Phase diagram determination}

A phase diagram is constituted of a binodal curve and tie lines. The determination of the binodal curve was carried out by a turbidimetric titration method [8]. Stock aqueous solutions of Ucon $40 \%(\mathrm{w} / \mathrm{w})$ and sodium citrate $25 \%(\mathrm{w} / \mathrm{w})$ of given $\mathrm{pHs}$ (5.20 or 8.20) were employed. Small aliquots of approximately $0.01-0.05 \mathrm{~g}$ of the polymer stock solution were added to $1 \mathrm{~g}$ of the sodium citrate stock solution, placed in a glass tube. After each aliquot addition, the system was thoroughly mixed. The first appearance of turbidity (the cloud point) indicated that the system was about to enter the two-phase area. With knowledge of the composition of the starting polymer and salt solutions and of the added amounts (grams), the total system composition, just prior to the two-phase formation, was calculated and provided a point on the binodal curve. The starting and added solution masses were measured on an analytical balance with a precision of $\pm 0.0001 \mathrm{~g}$. Additional binodal points were obtained by adding a small amount of water to clear the system and then enough drops of the Ucon stock solution to produce turbidity again. In order to obtain the binodal points corresponding to higher polymer concentration, the above mentioned procedure was inverted, thus titrating the stock polymer solution with the stock salt solution. The system temperature was maintained constant and controlled within $\pm 0.1^{\circ} \mathrm{C}$ by immersing the glass tube and the stock solutions in a thermostatic bath.

For the determination of the tie lines, a series of ATPSs of at least three different known total compositions were prepared in $5 \mathrm{~mL}$ graduated glass tubes (uncertainty $\pm 0.05 \mathrm{~mL}$ ) and placed in a thermostatic bath. After reaching the phaseequilibrium, visual estimates of top and bottom volumes ( $V_{\mathrm{T}}$ and $V_{\mathrm{B}}$, respectively) were made. When phases were separated, Ucon and citrate equilibrium concentrations were determined by an enzymatic assay and refractive index measurements in each phase.

The refractive index method [9] was used to determine the total concentrations of Ucon and citrate in the top and bottom equilibrium phases respectively. Standard curves (mass fraction up to $25 \%$ ) for refractive index of Ucon and sodium citrate (up to mass fraction $10 \%$ ) were determined using a refractometer ABBE, NAR 3T (Atago-Japan) with a precision of \pm 0.0001 . The determination was made at $25^{\circ} \mathrm{C}$ in a temperature controlled bath with an uncertainty of $\pm 0.1^{\circ} \mathrm{C}$. Since the refractive index of a polymer and salt solution at low total mass fraction (i.e. $<0.10$ ) is additive, a proper dilution of the phases was necessary to determine the refractive index. Since the citrate concentration can be measured accurately, the polymer concentration can be calculated by determining the refractive index of a phase and subtracting the contribution of the salt.

Citrate was determined by the following enzymatic assay. In a first step, citrate was converted to oxaloacetate and acetate in a reaction catalyzed by the enzyme citrate lyase (CL) [10]:

citrate $\stackrel{\mathrm{CL}}{\longrightarrow}$ oxaloacetate + acetate

In the presence of the enzymes L-malate dehydrogenase (L$\mathrm{MDH}$ ) and L-lactate dehydrogenase (L-LDH), oxaloacetate and its decarboxylation product pyruvate were reduced to L-malate and L-lactate, respectively, by reduced nicotinamide-adenine dinucleotide (NADH).

oxaloacetate $+\mathrm{NADH}+\mathrm{H}^{+\mathrm{L}-\mathrm{MDH}} \longrightarrow$ L-malate $+\mathrm{NAD}^{+}$

pyruvate $+\mathrm{NADH}+\mathrm{H}^{+} \stackrel{\mathrm{L}-\mathrm{LDH}}{\longrightarrow} \mathrm{L}$-lactate $+\mathrm{NAD}^{+}$

The amount of NADH oxidized in the above reactions is stoichiometric to the amount of citrate. NADH was determined by means of its light absorbance at $340 \mathrm{~nm}$. The uncertainty in the measurement of the mass fraction of the salt was estimated to be better than \pm 0.03 .

A mass balance check was made between the initial mass of each component and the amounts in the bottom and top phases on the basis of equilibrium compositions. The relative error in the mass balance was $<2 \%$. The tie-line lengths (TLL) for the different compositions were calculated according to:

$\mathrm{TLL}=\left[\left(w_{\mathrm{Ucon}}^{\mathrm{T}}-w_{\mathrm{Ucon}}^{\mathrm{B}}\right)^{2}+\left(w_{\mathrm{Cit}}^{\mathrm{T}}-w_{\mathrm{Cit}}^{\mathrm{B}}\right)^{2}\right]^{1 / 2}$

where $w_{\mathrm{Ucon}}^{\mathrm{T}}, w_{\mathrm{Cit}}^{\mathrm{T}}, w_{\mathrm{Ucon}}^{\mathrm{B}}, w_{\mathrm{Cit}}^{\mathrm{B}}$ are the top (T) and bottom (B) equilibrium mass fractions of Ucon and citrate. The tie line lengths are expressed in mass fractions.

\section{Results and discussion}

\subsection{Binodal curve}

The liquid-liquid equilibrium data of assayed Ucon/Cit ATPSs are given in Table 1. Fig. 1 shows the phase diagram corresponding to the Ucon/Cit $\mathrm{pH} 8.2$ ATPSs at $20^{\circ} \mathrm{C}$. It includes the binodal curve, which represents the borderline between the one-phase and the two-phase regions, and the tie lines. ATPSs 
Table 1

Phase compositions for Ucon/Cit ATPSs

\begin{tabular}{|c|c|c|c|c|c|c|c|c|c|}
\hline \multicolumn{2}{|c|}{ Total compositions } & \multicolumn{2}{|c|}{ Top phase } & \multicolumn{2}{|c|}{ Bottom phase } & STL* & $\sigma^{* *}$ & 100TLL & $K_{\mathrm{p}} * * *$ \\
\hline \multicolumn{10}{|l|}{$\mathrm{pH} 5.20$} \\
\hline 7.97 & 8.96 & 3.22 & 22.31 & 10.97 & 0.51 & -2.81 & 0.11 & 23.14 & 3.4 \\
\hline 8.99 & 8.98 & 2.81 & 25.88 & 12.12 & 0.39 & -2.74 & 0.08 & 27.14 & 4.3 \\
\hline 10.06 & 9.00 & 2.48 & 28.82 & 13.37 & 0.34 & -2.61 & 0.13 & 30.49 & 5.4 \\
\hline 5.70 & 10.65 & 3.32 & 21.40 & 7.28 & 3.48 & -4.53 & 0.14 & 18.35 & 2.2 \\
\hline 5.99 & 11.02 & 2.84 & 25.21 & 7.87 & 2.52 & -4.51 & 0.09 & 23.24 & 2.8 \\
\hline 6.22 & 17.08 & 1.89 & 33.40 & 10.64 & 0.42 & -3.77 & 0.08 & 34.12 & 5.6 \\
\hline 6.54 & 17.87 & 1.64 & 35.53 & 11.38 & 0.21 & -3.63 & 0.11 & 36.64 & 6.9 \\
\hline \multicolumn{10}{|c|}{$T=40^{\circ} \mathrm{C}$} \\
\hline \multicolumn{10}{|l|}{ pH 8.20} \\
\hline 6.45 & 9.44 & 1.95 & 22.73 & 9.62 & 0.09 & -2.95 & 0.09 & 23.90 & 4.9 \\
\hline 7.48 & 9.37 & 1.50 & 26.78 & 10.71 & 0.19 & -2.89 & 0.12 & 28.14 & 7.1 \\
\hline 8.47 & 9.40 & 1.12 & 30.39 & 11.80 & 0.19 & -2.83 & 0.11 & 32.03 & 10.5 \\
\hline 9.41 & 9.37 & 0.74 & 33.90 & 12.78 & 0.18 & -2.81 & 0.14 & 35.81 & 17.2 \\
\hline \multicolumn{10}{|c|}{$T=20^{\circ} \mathrm{C}$} \\
\hline 4.32 & 14.88 & 2.16 & 26.45 & 6.93 & 0.93 & -5.35 & 0.21 & 25.96 & 3.2 \\
\hline 4.49 & 16.33 & 1.93 & 29.37 & 7.58 & 0.61 & -5.09 & 0.15 & 29.31 & 3.9 \\
\hline 5.00 & 17.70 & 1.63 & 33.33 & 8.74 & 0.33 & -4.64 & 0.14 & 33.76 & 5.4 \\
\hline 5.49 & 19.18 & 1.41 & 36.24 & 10.02 & 0.22 & -4.18 & 0.17 & 37.03 & 7.1 \\
\hline \multicolumn{10}{|c|}{$T=40^{\circ} \mathrm{C}$} \\
\hline
\end{tabular}

*STL, tie line slope; $* * \sigma$, standard deviation; $* * * K_{\mathrm{p}}$ fraction of Cit retained in the bottom phase divided by the Cit in the top phase.

with total initial compositions above the binodal curve split into two equilibrium immiscible phases: a Ucon-poor, Cit-rich bottom layer and a Ucon-rich, Cit-poor upper phase. A similar behavior was observed for the traditional PEG/phosphate

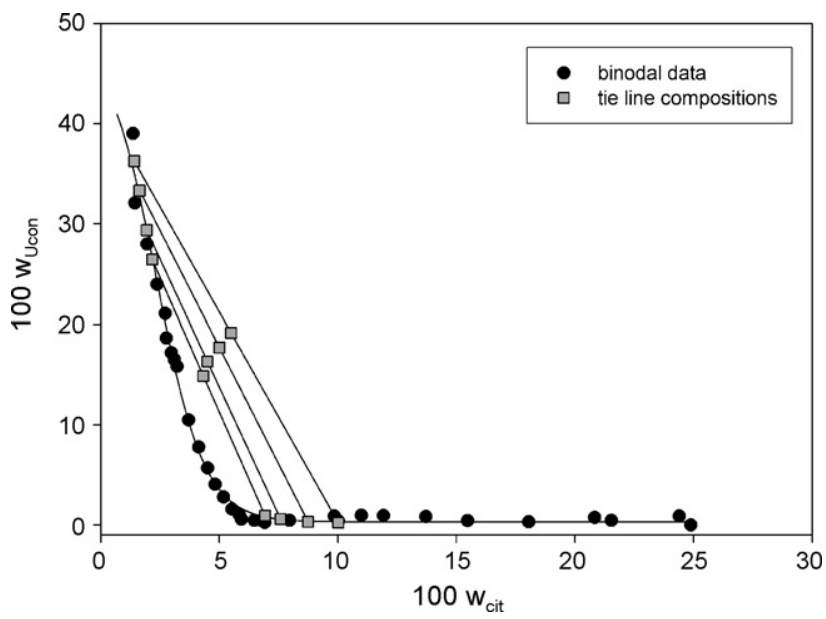

Fig. 1. Phase diagram of the Ucon/Cit pH 8.20 aqueous two-phase system. Temperature $20^{\circ} \mathrm{C}$.
ATPSs [1,2]. However, lesser salt and polymer concentrations are required for the formation of two phases for the Ucon/Cit ATPS when comparing with systems formed by PEG of similar molecular weight (4000) [2] due to the higher Ucon hydrophobic character. This makes the process more economical and reduces the environmental problems.

Different expressions have been proposed for the correlation of binodal data [11-13]. Table 2 shows the coefficients of determination $\left(R^{2}\right)$ obtained from fitting our experimental data with the most widely used empirical equations found in literature. Besides these empirical expressions, in a previous work [14], we have proposed the following sigmoidal equation:

$w_{\mathrm{Ucon}}=y_{0}+\frac{i}{1+\mathrm{e}^{-\left(w_{\mathrm{Cit}}-x_{0} / j\right)}}$

where $w_{\mathrm{Ucon}}$ and $w_{\mathrm{Cit}}$ (Ucon and Cit mass fraction, respectively) are the coordinates of the binodal points, $y_{0}, x_{0}, i$ and $j$, the fit parameters. The parameters of Eq. (2) along with the corresponding determination coefficients $\left(R^{2}\right)$ are summarized in Table 3. From visual inspection of Tables 2 and 3, it is possible to conclude that Eq. (2) is the most suitable to fit the binodal data 
Table 2

Fitting of binodal data by literature equations

\begin{tabular}{|c|c|c|c|c|}
\hline \multicolumn{2}{|c|}{ ATPSs } & \multicolumn{3}{|c|}{ Coefficient of determination $\left(R^{2}\right)$} \\
\hline \multirow[t]{2}{*}{$\mathrm{pH}$} & \multirow[t]{2}{*}{ Temperature $\left({ }^{\circ} \mathrm{C}\right)$} & \multicolumn{3}{|c|}{ Equations } \\
\hline & & $\mathrm{A}^{*}$ & $\mathrm{~B}^{* *}$ & $\mathrm{C}^{* * *}$ \\
\hline \multirow[t]{3}{*}{5.2} & 5 & 0.746 & 0.963 & 0.745 \\
\hline & 20 & 0.825 & 0.917 & 0.832 \\
\hline & 40 & 0.979 & 0.995 & 0.990 \\
\hline \multirow[t]{3}{*}{8.2} & 5 & 0.791 & 0.978 & 0.793 \\
\hline & 20 & 0.628 & 0.898 & 0.682 \\
\hline & 40 & 0.959 & 0.985 & 0.823 \\
\hline
\end{tabular}

* Equation A [11]: $1 / w_{\mathrm{Cit}}=a+b w_{\mathrm{Ucon}}^{0.5}+c w_{\mathrm{Ucon}} ; a, b, c$ fitting parameters.

** Equation B [12]: $w_{\mathrm{Ucon}}=a+b w_{\mathrm{Cit}}^{0.5}+c w_{\mathrm{Cit}} ; a, b, c$ fitting parameters.

*** Equation $\mathrm{C}$ [13]: $w_{\mathrm{Cit}}=a \ln \left(w_{\mathrm{Ucon}}+c\right)+b ; a, b, c$ fitting parameters.

since their $R^{2}$ values are closer to one than those obtained from literature expressions.

The effect of $\mathrm{pH}$ on phase separation was evaluated. Fig. 2(A and B) summarizes the binodal data corresponding to ATPSs of Ucon/Cit at different pHs. Binodal curves show similar shapes for the several $\mathrm{pH}$ values and the two-phase area is found to be expanded when $\mathrm{pH}$ is increased. At $20^{\circ} \mathrm{C}$, binodal curves corresponding to the different $\mathrm{pHs}$ tend to superimpose at high concentrations of Ucon or Cit, thus indicating that either the exclusion or the salting out effect respectively prevails in phaseseparation processes. When Ucon and Cit concentrations adopt intermediate values, a smaller concentration of citrate is needed for two-phase formation at basic $\mathrm{pHs}$ (with higher ratios between trivalent and divalent citrate ions). This effect is also observed in binodal curves at $5{ }^{\circ} \mathrm{C}$ (Fig. 2A), even at high Ucon concentrations (low Cit concentrations) at which no superimposition of binodal curves is observed. At low temperatures, the EO and PO units in the copolymer, are known to be strongly hydrated with two or three water molecules [7]. Similarly, ionic species in solution are known to be hydrated and the extent of hydration depends upon the ion valency. Thus, triply charged citrate can be expected to be more effective than doubly charged citrate in salting out the copolymer because of competition for water. Therefore, trivalent ions are more efficient than divalent ions in promoting the phase separation [15]. However, this effect does not seem to be significant at low Cit concentration at $20^{\circ} \mathrm{C}$, since binodal curves corresponding to the different $\mathrm{pHs}$ overlap. In this case, the breakdown of structured water molecules around

Table 3

Values of fit parameters $i, j, x_{0}$ and $y_{0}$ of Eq. (2) for Ucon/Cit ATPSs

\begin{tabular}{|c|c|c|c|c|c|c|}
\hline \multicolumn{2}{|c|}{ ATPSs } & \multicolumn{5}{|c|}{ Parameters } \\
\hline$\overline{\mathrm{pH}}$ & Temperature $\left({ }^{\circ} \mathrm{C}\right)$ & $i$ & $j$ & $x_{0}$ & $y_{0}$ & $R^{2}$ \\
\hline \multirow[t]{3}{*}{5.2} & 5 & 51.63 & -1.48 & 2.79 & 0.30 & 0.992 \\
\hline & 20 & 65.24 & -1.88 & 2.01 & -0.23 & 0.995 \\
\hline & 40 & 159.4 & -2.77 & -1.27 & -2.60 & 0.996 \\
\hline \multirow[t]{3}{*}{8.2} & 5 & 57.44 & -1.54 & 1.31 & -0.14 & 0.991 \\
\hline & 20 & 48.45 & -1.01 & 2.35 & 0.30 & 0.992 \\
\hline & 40 & 1114 & -0.81 & -2.16 & 1.05 & 0.989 \\
\hline
\end{tabular}

$R^{2}$, coefficient of determination.
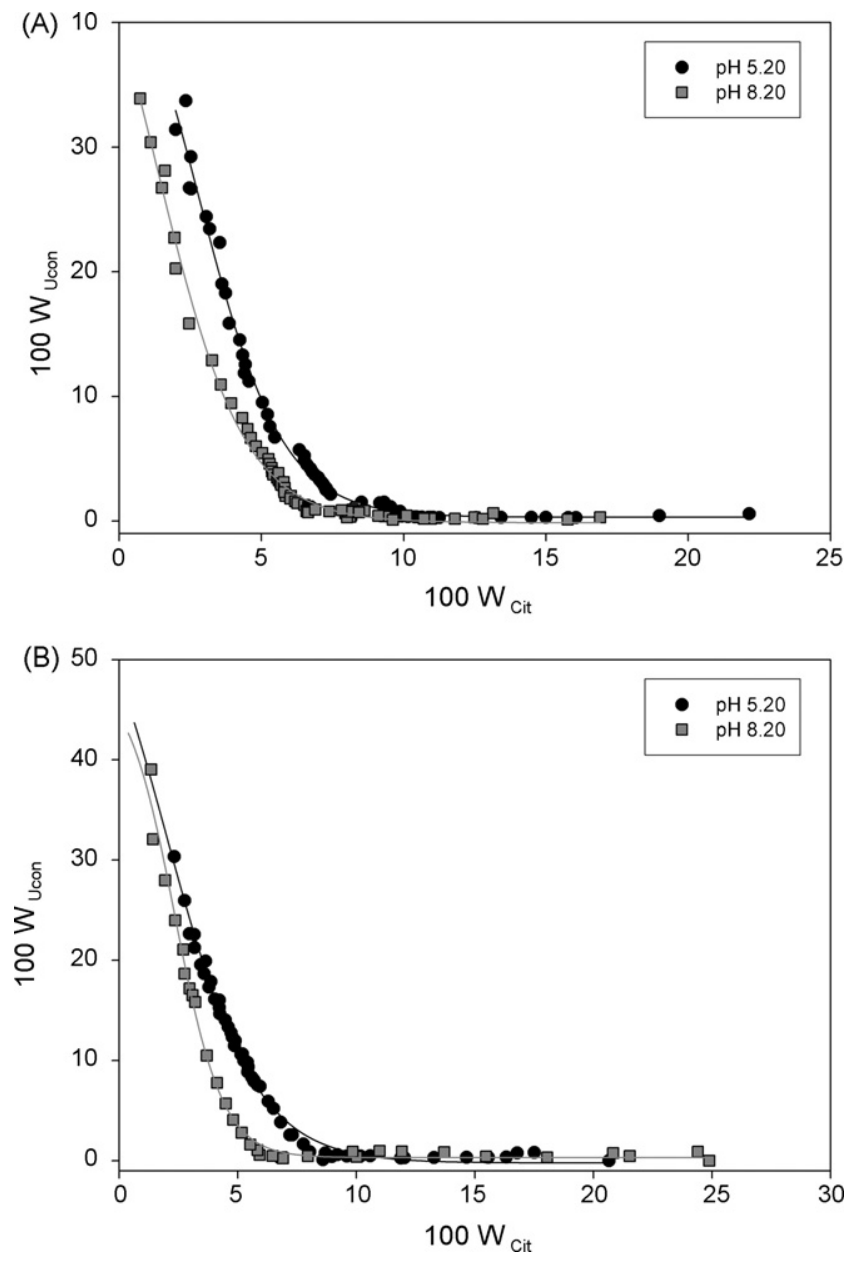

Fig. 2. Effect of $\mathrm{pH}$ on binodal curve of Ucon/Cit aqueous two-phase systems. (A) $5{ }^{\circ} \mathrm{C}$; (B) $20^{\circ} \mathrm{C}$.

the EO/PO-chains associated with an increase in temperature, is probably the predominant cause of phase separation.

The effect of temperature on phase-separation processes is given in Fig. 3. An increase in temperature from 5 to $20^{\circ} \mathrm{C}$ induced a slight increase in the biphasic area, while a significant

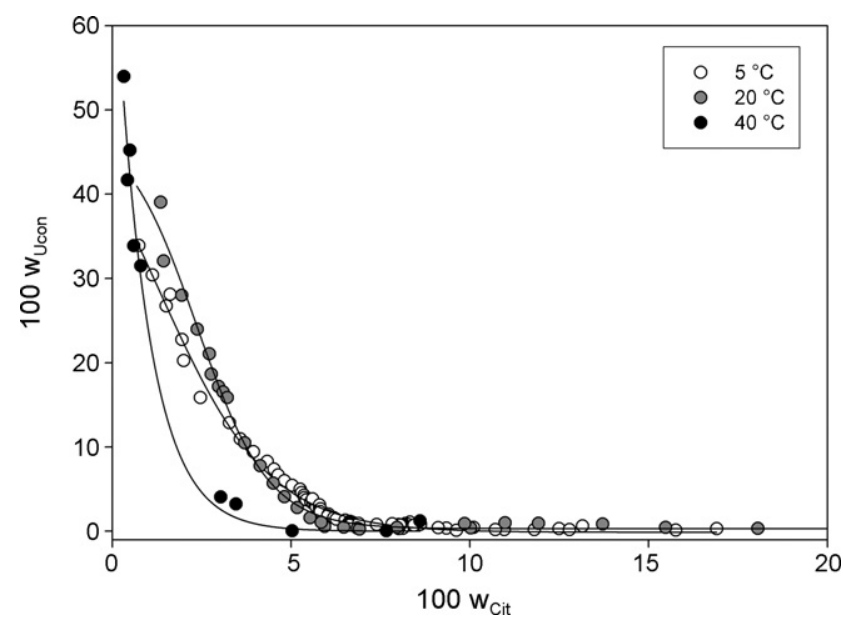

Fig. 3. Effect of temperature on binodal curve of Ucon/Cit pH 8.20 aqueous two-phase system. 
expansion of the two-phase region was observed when the temperature was raised up to $40^{\circ} \mathrm{C}$. This trend was also observed for other polymer/salt systems such as PEG/Cit [14]. According to the model proposed by Kjellander and Florin [16], the entropically unfavorable structuring of water produced by Ucon at low temperatures is overcome owing to the large decrease in enthalpy (due to the energetically favorable and highly directional interactions, such as hydrogen-bonding, between unlike molecules). At higher temperatures, provided that the structure of water in the Ucon hydration shell does not break down too rapidly with increasing temperature [17], the unfavorable entropy contribution becomes prominent and the system phase separates itself. In addition by increasing the temperature the magnitude of the tie line slopes increases (see Table 1), indicating the increase of the asymmetry of the diagrams. Similar results were obtained for other polymer/salt systems from literature [18].

\subsection{Tie lines}

The equilibrium data are listed in Table 1. It also lists the value of the partition coefficient $\left(K_{\mathrm{p}}\right)$ of the salt, defined as the fraction of salt retained in the bottom phase divided by the salt in the top phase. High values of $K_{\mathrm{p}}$ were obtained (from 2.2 to 22.6) which is an indication of the separation obtained by adding an amount of Ucon to a given brine solution.

For most of the assayed systems, the tie lines became steeper for total compositions in the vicinity of the critical point. An increase in the TLS magnitude indicates an increase in the difference between the polymer concentrations at a given difference in the salt concentrations in the same phase. This implies a decrease in the mutual solubility of the aqueous polymer- and salt-containing media. A similar behavior was observed for other ATPSs [19].

Empirical equations have been proposed to ascertain the reliability of calculated tie-line data in traditional liquid-liquid extraction, being the most widely used those of Othmer-Tobias (Eq. (3)) and Bancroft (Eq. (4)) [20]:

$$
\begin{aligned}
& \frac{1-w_{\mathrm{Ucon}}^{\mathrm{T}}}{w_{\mathrm{Ucon}}^{\mathrm{T}}}=k\left(\frac{1-w_{\mathrm{Cit}}^{\mathrm{B}}}{w_{\mathrm{Cit}}^{\mathrm{B}}}\right)^{n} \\
& \frac{w_{\mathrm{H}_{2} \mathrm{O}}^{\mathrm{B}}}{w_{\mathrm{Cit}}^{\mathrm{B}}}=k_{1}\left(\frac{w_{\mathrm{H}_{2} \mathrm{O}}^{\mathrm{T}}}{w_{\mathrm{Ucon}}^{\mathrm{T}}}\right)^{r}
\end{aligned}
$$

Table 4

Values of the parameters of Othmer-Tobias and Bancroft equations, $k, n, k_{1}$ and

\begin{tabular}{|c|c|c|c|c|c|c|c|}
\hline \multicolumn{2}{|c|}{ ATPSs } & \multicolumn{6}{|c|}{ Parameters } \\
\hline$\overline{\mathrm{pH}}$ & $\overline{\text { Temperature }\left({ }^{\circ} \mathrm{C}\right)}$ & $\bar{k}$ & $n$ & $R^{2}$ & $k_{1}$ & $r$ & $R^{2}$ \\
\hline \multirow[t]{3}{*}{5.2} & 5 & 0.178 & 1.412 & 0.992 & 3.452 & 0.713 & 0.991 \\
\hline & 20 & 0.110 & 1.361 & 0.985 & 5.312 & 0.688 & 0.982 \\
\hline & 40 & 0.023 & 1.767 & 0.999 & 8.483 & 0.562 & 0.999 \\
\hline \multirow[t]{3}{*}{8.2} & 5 & 0.071 & 1.728 & 0.999 & 4.609 & 0.596 & 0.999 \\
\hline & 20 & 0.144 & 1.132 & 0.987 & 5.657 & 0.879 & 0.985 \\
\hline & 40 & 0.014 & 1.570 & 0.981 & 14.969 & 0.608 & 0.980 \\
\hline
\end{tabular}
$r$ for Ucon/Cit ATPSs

$R^{2}$, coefficient of determination. where $w_{\mathrm{H}_{2} \mathrm{O}}^{\mathrm{T}}$ and $w_{\mathrm{H}_{2} \mathrm{O}}^{\mathrm{B}}$ are the mass fractions of water in the top and bottom phases; $k, n, k_{1}$ and $r$ represent the parameters to be determined. Linearization of both equations produced acceptable consistency in the results. Values of the fit parameters and the corresponding coefficients of determination are given in Table 4.

\section{Conclusions}

The phase diagrams of Ucon/Cit ATPSs were determined. Reliable and complete data on the composition and properties of these systems were not available at present, being this information necessary for the design of an extraction process. The phase formation proved to be both temperature and $\mathrm{pH}$-dependent. Much lower amounts of polymer and salt than conventional systems are required to form the two phases, thus reducing the environmental impact. In addition, thermo-separating properties of Ucon can be used to recycle this polymer from the polymer rich-phase. These characteristics and several additional advantages such as biodegradability of citrate anion, low-cost and rapid phase separation make Ucon/Cit ATPSs a promising, versatile and attractive system in the field of bio-separation.

\section{Acknowledgments}

This work was supported by a grant from CONICET, and ALFA II-0440-FA-Exchange programme between Universities of the European Union and Latin America ("Valorisation of natural resources: obtention of high valuables compounds through extraction and/or biotransformation for application in novel and functional foods, for their use in diagnostics and for novel industrial applications, VALNATURA").

We thank Maria Robson and Marcela Culasso, Mariana de Sanctis and Geraldine Raimundo for the language correction of this manuscript.

\section{References}

[1] P.Å. Albertsson, Partition of Cell Particles and Macromolecules, 2nd ed., John Wiley and Sons, New York, 1971.

[2] B.Y. Zaslavsky, Aqueous Two-phase Partitioning Physical Chemistry and Bioanalytical Applications, Marcel Dekker, Inc., New York, 1994.

[3] G. Reh, B.B. Nerli, G.A. Picó, Isolation of alpha-1-antitrypsin from human plasma by partitioning in aqueous biphasic systems of polyethyleneglycol-phosphate, J. Chromatogr. B. 780 (2002) 389-396.

[4] D.P. Harris, A.T. Andrews, G. Whright, D.L. Pyle, J.A. Asenjo, The application of aqueous two-phase systems to the purification of pharmaceutical proteins from transgenic sheep milk, Bioseparation 7 (1997) 31-37.

[5] T. Murugesan, M. Perumalsamy, Liquid-liquid equilibria of poly(ethyleneglycol) 2000 + sodium citrate + water at $(25,30,35$, 40, and $45^{\circ} \mathrm{C}$ ), J. Chem. Eng. Data 50 (2000) 1392-1395.

[6] M. Rito-Palomares, Practical application of aqueous two-phase partition to process development for the recovery of biological products, J. Chromatogr. B 807 (2004) 3-11.

[7] J. Persson, H.O. Johanson, I. Galaev, B. Mattiasson, F. Tjerneld, Aqueous polymer two-phase systems formed by new thermoseparating polymers, Bioseparation 9 (2000) 105-116.

[8] R. Hatti-Kaul, Methods in Biotechnology. Aqueous two-phase systems. Methods and Protocols, Humana Press, Inc., New Jersey, 2000. 
[9] Partitioning in Aqueous Two-phase Systems, in: H. Walter, D. Brooks, D. Fisher (Eds.), Theory, Methods, Uses, and Applications to Biotechnology, Academic Press, Inc., Orlando, 1985.

[10] H. Mollering, W. Gruber, Determination of citrate with citrate lyase, Anal. Biochem. 17 (1966) 369-376.

[11] T.A. Graber, M.E. Taboada, Liquid-liquid equilibrium of the poly(ethylene glycol) + sodium nitrate + water system at 298.15 K, J. Chem. Eng. Data 45 (2000) 182-184.

[12] M.T. Zafarani-Moattar, A.A. Hamidi, Liquid-liquid equilibria of aqueous two-phase poly(ethyleneglycol)-potassium citrate system, J. Chem. Eng. Data 48 (2003) 262-265.

[13] P. González Tello, F. Camacho, G. Blázquez, F.J. Alarcón, Liquid-liquid equilibrium in the system poly(ethyleneglycol) $+\mathrm{MgSO}_{4}+\mathrm{H}_{2} \mathrm{O}$ at $298 \mathrm{~K}$, J. Chem. Eng. Data 41 (1996) 1333-1336.

[14] G. Tubio, L. Pellegrini, B.B. Nerli, G.A. Picó, Liquid-liquid equilibria of aqueous two-phase systems containing poly(ethyleneglycols) of different molecular weight and sodium citrate, J. Chem. Eng. Data 51 (2006) 209-212.

[15] K. Mishima, K. Nakatani, T. Nomiyama, K. Matsuyama, M. Nagatani, H. Nishikawa, Liquid-liquid equilibria of aqueous two-phase systems contain- ing polyethylene glycol and dipotassium hydrogenphosphate, Fluid Phase Equilibria 107 (2001) 269-276.

[16] R. Kjellander, E. Florin, Water structure and changes in thermal stability of the system poly(ethylene oxide)-water, J. Chem. Soc. Faraday Trans. 1 77 (1981) 2053.

[17] M. Harris, Poly(ethylene glycol) Chemistry: Biotechnical and Biomedical Applications, Plenum Press, New York, 1992.

[18] M.T. Zafarani-Moattar, R. Sadeghi, A.A. Hamidi, Liquid-liquid equilibria of an aqueous two-phase system containing polyethylene glycol and sodium citrate: experiment and correlation, Fluid Phase Equilibria 219 (2004) 147-153.

[19] M. Pereira, Y.T. Wu, P. Madeira, A. Venâncio, E. Macedo, J. Teixeira, Liquid-liquid equilibrium phase diagrams of new aqueous two-phase systems: ucon 50-HB5100 + ammonium sulfate + water, ucon 50-HB5100 + poly(vinyl alcohol) + water, 50-HB5100 + hydroxypropyl starch + water, and pol(ethylene glycol) $8000+$ poly (vinyl alcohol) + water, J. Chem. Eng. Data 49 (2004) 43-47.

[20] D.F. Othmer, P.E. Tobias, Liquid-liquid extraction data-toluene and acetaldehyde systems, Ind. Eng. Chem. 34 (1942) 690-692. 